Article

\title{
Bio-Based Composites with Enhanced Matrix-Reinforcement Interactions from the Polymerization of $\alpha$-Eleostearic Acid
}

\author{
Amanda Murawski and Rafael L. Quirino* \\ Chemistry Department, Georgia Southern University, Statesboro, GA 30458, USA \\ * Correspondence: rquirino@georgiasouthern.edu
}

Received: 25 June 2019; Accepted: 9 July 2019; Published: 17 July 2019

check for updates

\begin{abstract}
Vegetable oil-based composites have been proposed as interesting bio-based materials in the recent past. The carbon-carbon double bonds in unsaturated vegetable oils are ideal reactive sites for free radical polymerization. Without the presence of a reinforcement, typical vegetable oil-based polymers cannot achieve competitive thermo-mechanical properties. Compatibilizers have been utilized to enhance the adhesion between resin and reinforcement. This work discusses the antagonist implications of polarity and crosslink density of an unprecedented polar $\alpha$-eleostearic acid-based resin reinforced with $\alpha$-cellulose, eliminating the need of a compatibilizer. It is shown that the polar regions of $\alpha$-eleostearic acid can interact directly with the polar reinforcement. The successful isolation of $\alpha$-eleostearic acid from tung oil was verified via GC-MS, ${ }^{1} \mathrm{H}$ NMR, Raman, and FT-IR spectroscopies. The optimal cure schedule for the resin was determined by DSC and DEA. The composites' thermo-mechanical properties were assessed by TGA, DSC, and DMA.
\end{abstract}

Keywords: bio-based materials; fatty acids; thermosets; tung oil; cellulose composites

\section{Introduction}

In the past decade, vegetable oils have received a lot of attention as an attractive bio-renewable starting material for the preparation of bio-based polymers. Polymers prepared from vegetable oils have the advantage of being versatile due to their easily tunable properties, which can be simply adjusted with changes in the resin composition. Applications in biomedicine, construction, furniture, adhesives, and coatings can benefit from the development of vegetable oil-based polymers and composites. It is widely accepted that fossil fuels are limited and non-renewable in practical terms [1]. It is, therefore, imperative to continue research for more sustainable alternatives in order to alleviate economic stress and petroleum depletion impacts in the polymer and composites industry. Furthermore, the development of bio-renewable alternatives offers materials with the potential of exhibiting a positive environmental impact, especially with respect to current waste disposal concerns.

Polyurethanes [2,3], polyester amides [4], polyolefins [5], and alkyd resins [6] are just some of the current resins on the market that can be prepared from vegetable oils. In order to improve mechanical properties, these resins have been reinforced with inorganic fillers, such as clays [7]. The difficulty with binding between vegetable oil-based polymers and reinforcement lies within typical incompatibility of common hydrophobic resins and hydrophilic fillers. In vegetable oil-based thermosets reinforced with rice hulls [8], and wood flour [9], mechanical property improvements have been made by enhancing the reinforcement-resin interactions with the addition of maleic anhydride as a co-monomer. In another study, asolectin, a phospholipid mixture isolated from soybeans previously used as a synthetic protein transport vessel $[10,11]$, was employed as a bio-based compatibilizer between cellulose and a tung oil-based matrix [12]. The amphiphilic nature of asolectin, with long unsaturated carbon chains 
and a highly polar phosphate group, ensures its role as an ideal bio-based compatibilizer. Indeed, an enhancement of the thermo-mechanical properties was observed with the addition of asolectin to tung oil/cellulose composites [12].

Industrially, vegetable oils find use in biofuels, drying agents, food, cosmetics, soap and detergents, and pharmaceuticals [13]. The chemical structure of vegetable oils consists of a triglyceride, which corresponds to glycerol esters connected to fatty acids. The carbon-carbon double bonds in unsaturated vegetable oils are ideal reactive sites for the chemical industry, offering a chemical handle for modifications leading to a variety of bio-based precursors [14]. Most unsaturated oils must be modified before free radical polymerization due to the low reactivity of non-conjugated carbon-carbon double bonds towards free radicals. In tung oil, approximately $83 \%$ of the fatty acid chains are $\alpha$-eleostearic acid, a naturally triply-conjugated fatty acid. The naturally conjugated carbon-carbon double bonds in tung oil (at carbons 9-cis, 11-trans, and 13-trans) [15] can readily react with free radicals and polymerize in the presence of oxygen [14]. The co-polymerization of tung oil with vinyl co-monomers via cationic, thermal, or free radical polymerizations results in rigid, crosslinked polymers [16]. Other studies have further shown that a biopolymer can successfully be prepared from tung oil by cationic polymerization for self-healing applications in the curing of micro-cracks [17].

Due to the reactivity of tung oil and the promising thermo-mechanical properties obtained from tung oil-based/cellulose composites [12], it was hypothesized that an improvement in the compatibility of the matrix and the reinforcement can be obtained, without compromising the bio-based nature of the system, by using tung oil fatty acids as the major resin component. Cellulose is a hydrophilic polysaccharide composed of repeating D-glucose units linked through $\beta$-(1-4)-glycosidic bonds [18]. Due to celluloses' biodegradability, accessibility, and mechanical properties, it has become increasingly popular for the production of low-cost materials.

The work described in this manuscript proposes a bio-based composite system in which the need of a compatibilizer between the hydrophilic cellulose and a tung oil-based matrix is eliminated. By isolating the fatty acid, $\alpha$-eleostearic acid, from tung oil, and subsequently polymerizing it, a polymeric network containing carboxyl groups with potential to hydrogen bond with polar reinforcements, such as cellulose, is obtained. It is suggested that intermolecular hydrogen bonding between the tung oil fatty acid and cellulose creates an interlocking polymer network, therefore enhancing the thermo-mechanical properties. The successful isolation of fatty acids in this work was verified via GC-MS and ${ }^{1} \mathrm{H}$ NMR spectroscopy. Additionally, all materials were analyzed by Raman, and FT-IR spectroscopies. The polymerization of the resins was monitored by dielectric analysis (DEA) to obtain an optimal cure schedule. The morphology of the composites prepared was verified by scanning electron microscopy (SEM), and their hydrophilicity was assessed by means of contact angle and water absorption experiments. The final materials' thermo-mechanical properties were assessed by thermogravimetric analysis (TGA), differential scanning calorimetry (DSC), and dynamic mechanical analysis (DMA). When comparing cellulose composites prepared from tung oil fatty acids and tung oil methyl esters, it was found that the former exhibit higher binding affinity between matrix and reinforcement, which is reflected in their thermo-mechanical properties. The results presented here confirm the initial hypothesis that a better fiber-matrix interaction may be obtained with the use of fatty acids as co-monomers for the fabrication of biocomposites at the expense of a lower crosslink density when compared to tung oil triglycerides.

\section{Experimental}

\subsection{Materials}

n-butyl methacrylate (BMA), tung oil (TO), $\alpha$-cellulose, and di-tert-butyl peroxide (DTBP) were purchased from Sigma-Aldrich (St. Louis, MO, USA). Methanol and acetone were procured from BDH Analytical Chemicals (Radnor, PA, USA). 50\% w/w sodium hydroxide aqueous solution ( $\mathrm{NaOH}$ ), 38.0\% $w / w$ hydrochloric acid aqueous solution $(\mathrm{HCl})$, and chloroform were obtained from Fisher Chemical 
(Fair Lawn, NJ, USA). Deuterated chloroform (chloroform-D) was purchased from Cambridge Isotope Laboratories, Inc. (Andover, MA, USA). Divinylbenzene (DVB) (composed of meta, para and ortho isomers) was procured from TCI America (Portland, OR, USA).

\subsection{Methods}

\subsubsection{Fatty Acid Isolation from Tung Oil}

The saponification reaction used in this work was adapted from the literature as follows [17]. A 50\% $w / w$ sodium hydroxide aqueous solution was diluted to $20 \% w / w$ with deionized water. Two-hundred milliliters of the $20 \%$ sodium hydroxide solution was added to $100.0 \mathrm{~g}$ of tung oil, and the mixture was kept in a water bath under agitation with a magnetic stir bar at $70{ }^{\circ} \mathrm{C}$ for $1 \mathrm{~h}$. Three-hundred milliliters of water at $70^{\circ} \mathrm{C}$ were then added and the mixture was left undisturbed for $30 \mathrm{~min}$ before an additional $300.0 \mathrm{~mL}$ of water at $70{ }^{\circ} \mathrm{C}$ was added. After a final addition of $95.0 \mathrm{~mL}$ of the $\mathrm{HCl}$ solution (38\%), the mixture was kept under agitation at $70{ }^{\circ} \mathrm{C}$ for $1 \mathrm{~h}$. The precipitates formed were filtered and washed with $200 \mathrm{~mL}$ of deionized water, followed by $300 \mathrm{~mL}$ of methanol. The filtered material was then dried overnight at $70{ }^{\circ} \mathrm{C}$ under vacuum.

\subsubsection{Transesterification of Tung Oil}

The procedure for the transesterification of tung oil with methanol was adapted from the literature and is described herein as follows [19]. Twenty grams of tung oil was refluxed with $30.0 \mathrm{~mL}$ of methanol in the presence of $0.5 \mathrm{~g}$ of $50 \%$ sodium hydroxide aqueous solution for $2 \mathrm{~h}$. The reaction mixture was then decanted in a separatory funnel overnight. After phase separation, the bottom layer, containing glycerol and other aqueous by-products, was discarded. The upper layer, containing the methyl esters, was analyzed by proton nuclear magnetic resonance spectroscopy $\left({ }^{1} \mathrm{H}\right.$ NMR) and gas chromatography (GC) for purity and verification of successful isolation.

\subsubsection{Preparation of Thermosetting Resins}

Fatty acid resins were prepared by heating $5.0 \mathrm{~g}$ of the fatty acids obtained from tung oil, $3.0 \mathrm{~g}$ of $\mathrm{BMA}$, and $2.0 \mathrm{~g}$ of DVB in a capped $20 \mathrm{~mL}$ scintillation vial at $50{ }^{\circ} \mathrm{C}$ until the fatty acid was completely molten. The mixture was then vortexed for $30 \mathrm{~s}$ before $0.5 \mathrm{~g}$ of DTBP was introduced. The mixture was vortexed again for an additional $30 \mathrm{~s}$. The scintillation vial was then placed in a convection oven for the designated temperatures and times, as discussed in the text. Thermosetting resins from tung oil and from tung oil methyl esters were prepared following an identical procedure, with the exception of the initial heating at $50{ }^{\circ} \mathrm{C}$. Figure $\mathrm{S} 1$ shows a simplified free radical polymerization reaction mechanism for $\alpha$-eleostearic acid. BMA and DVB were not included in the reaction mechanism presented in Figure S1 for clarity reasons.

\subsubsection{Preparation of Cellulose-Reinforced Composites}

The aforementioned thermosetting resins were reinforced with $\alpha$-cellulose by saturating the monomer mixture with $3.0 \mathrm{~g}(23 \mathrm{wt} \%)$ of the reinforcement prior to the addition of $0.5 \mathrm{~g}$ of DTBP. The solution was vortexed prior to cure in a convection oven (time and temperature cure schedules were evaluated and confirmed by DEA, DSC, and Raman spectroscopy, as discussed in Section 3.2). Figure S2 displays a general schematic for the preparation of fatty acid-based composites.

\subsection{Characterization}

Fourier-transformed infrared (FTIR) spectra were collected using a Thermo Nicolet Avatar 370-FTIR spectrometer (Thermo Scientific, Waltham, MA, USA) with an attenuated total reflectance (ATR) accessory. The spectra were obtained in the $400-4000 \mathrm{~cm}^{-1}$ spectral region, with 64 scans, and $4 \mathrm{~cm}^{-1}$ resolution. Proton nuclear magnetic resonance $\left({ }^{1} \mathrm{H}\right.$ NMR) spectra were obtained on an Agilent MR400DD2 spectrometer (Santa Clara, CA, USA) operating at $400 \mathrm{MHz}$. Raman spectra were 
collected on a DXR Raman Microscope (Thermo Scientific, Waltham, MA, USA) at a laser power of $1 \mathrm{~mW}$.

Gas-chromatography/mass spectrometry (GC-MS) analyses were completed on a Shimadzu GC-17A gas chromatograph equipped with a mass spectrometer detector Shimadzu GCMS-QP5050A (Kyoto, Japan) using a $30 \mathrm{~m}$ Rxi-5ms column (Restek, Bellefonte, PA, USA) with an internal diameter of $0.25 \mathrm{~mm}$ and a film thickness of $0.25 \mu \mathrm{m}$. Samples were dissolved in acetone, leading to an approximate concentration of $5 \%(v / v)$. Helium was used as the carrier gas at a total (column + split) flow rate of $10 \mathrm{~mL} / \mathrm{min}$. The temperature was initially held at $70^{\circ} \mathrm{C}$ for $2 \mathrm{~min}$, then increased to $300^{\circ} \mathrm{C}$ at a heating rate of $15^{\circ} \mathrm{C} / \mathrm{min}$, and finally held at $300^{\circ} \mathrm{C}$ for $13 \mathrm{~min}$.

Dielectric analysis (DEA) measurements were completed on an Epsilon DEA 230/1 cure monitor (Netzsch Instruments North America LLC, Burlington, MA, USA). Differential scanning calorimetry (DSC) studies were conducted on a Q250 DSC instrument from TA Instruments (New Castle, DE, USA). 5-10 mg of sample were heated from -30 to $200{ }^{\circ} \mathrm{C}$ at a rate of $10{ }^{\circ} \mathrm{C} / \mathrm{min}$ under $\mathrm{N}_{2}$ atmosphere in aluminum hermetic pans.

Static and dynamic contact angles were measured using a goniometer (Ramé-hart, Succasunna, NJ, USA) equipped with an automatic drop dispenser. For the static contact angle measurements, $2.0 \mu \mathrm{L}$ of water were dispensed onto a flat surface of the sample and the contact angle was measured every $30 \mathrm{~s}$ for a total of 10 measurements, and repeated for a total of three trials. The average angle from the three trials are reported. For the dynamic contact angle, measurements were generated by slowly enlarging the droplet size (advancing contact angle, $\theta_{\mathrm{a}}$ ) and then retracting it (receding contact angle, $\theta_{\mathrm{r}}$ ). For each expanding or retraction step, $0.25 \mu \mathrm{L}$ of water was used.

Water absorption experiments were conducted by weighing fully cured samples at their dry state $\left(W_{\mathrm{d}}\right)$, and then submerging them in water for $24 \mathrm{~h}$. After $24 \mathrm{~h}$, the samples were removed from water and their wet weight $\left(W_{\mathrm{w}}\right)$ was recorded after gently drying the excess water with a kimwipe. The morphology analyses were completed on a JSM-760F field emission scanning electron microscope (SEM) (JEOL, Peabody, MA, USA), using an accelerating voltage of $1.0 \mathrm{kV}$, secondary electron imaging (SEI) and lower detector scanning (LEI) modes, and an emission current of approximately $81 \mathrm{~mA}$. All samples were sputter-coated with gold prior to imaging.

Thermal degradation profiles were obtained on a Q50 thermal gravimetric analyzer (TA Instruments) using samples of approximately $10 \mathrm{mg}$. The experiments were run under air, from 30 to $500{ }^{\circ} \mathrm{C}$, with a heating rate of $10^{\circ} \mathrm{C} / \mathrm{min}$. Thermo-mechanical properties were assessed on a Q800 DMA (TA Instruments) using a three-point bending fixture with $15 \mathrm{~mm}$ gap. Rectangular samples $(1.5 \mathrm{~mm} \times 10.0 \mathrm{~mm} \times 17.0 \mathrm{~mm}$-thickness $\times$ width $\times$ length $)$ were cut and subjected to an iso-strain experiment with an amplitude of $14 \mu \mathrm{m}$. The temperature was increased from -60 to $150{ }^{\circ} \mathrm{C}$ at a heating rate of $3{ }^{\circ} \mathrm{C} / \mathrm{min}$.

\section{Results and Discussion}

\subsection{Starting Materials}

Tung oil and its corresponding fatty acids and methyl esters were analyzed by FTIR, ${ }^{1} \mathrm{H}$ NMR, and Raman spectroscopies, and by GC-MS in order to check for purity and to confirm the structure of the bio-based starting materials used for the preparation of polymers and composites in this work. When comparing the FTIR spectra of tung oil, isolated $\alpha$-eleostearic acid, and tung oil methyl esters (Figure 1), it can be observed that the main structural features of the fatty acid chain in tung oil are unaltered after saponification and transesterification. Signals, such as the strong peak at approximately $1750 \mathrm{~cm}^{-1}$, representing the carbonyl $\mathrm{C}=\mathrm{O}$ stretch, and the strong peak at approximately $1000 \mathrm{~cm}^{-1}$, which corresponds to the $\mathrm{C}-\mathrm{O}$ stretch in the ester (tung oil and methyl esters) or carboxyl ( $\alpha$-eleostearic acid) functional groups, can be seen in all three spectra (Figure 1). Likewise, the peak at $990-980 \mathrm{~cm}^{-1}$, representing the $\mathrm{C}-\mathrm{H}$ wagging vibrations of conjugated carbon-carbon double bonds are also observed in all three compounds (Figure 1) [20]. It's interesting to note the absence of the typical signal for the 
$\mathrm{O}-\mathrm{H}$ stretch in the spectrum of isolated $\alpha$-eleostearic acid (Figure 1). It is believed that, due to the basic reaction conditions employed during saponification, the final fatty acid is obtained in its salt form as a sodium carboxylate, which does not affect its subsequent polymerization and/or future interactions with the reinforcement.

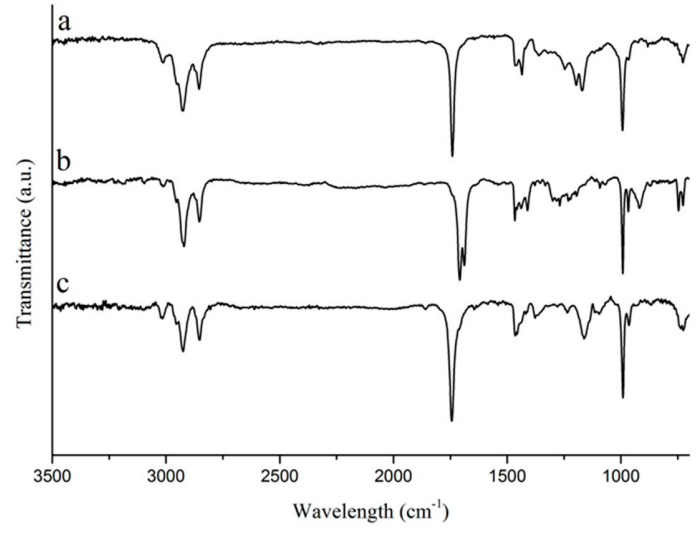

Figure 1. FTIR spectra of (a) tung oil methyl esters, (b) isolated $\alpha$-eleostearic acid, and (c) tung oil.

The ${ }^{1} \mathrm{H}$ NMR spectra of the bio-based starting materials (Figure 2) and the peak integration of their corresponding key signals provide further insight into reaction completion and product purity. The characteristic signals for the methylene (4.1-4.3 ppm, signal 6) and the methyne ( 5.25 ppm, signal 7) hydrogens of the glycerol backbone can be clearly seen in the ${ }^{1} \mathrm{H}$ NMR spectrum of tung oil (Figure 2a). Their absence from the spectra of tung oil methyl esters and isolated $\alpha$-eleostearic acid (Figure 2b,c, respectively) confirms successful separation of any residual glycerol from the desired products. The intense singlet at $3.5 \mathrm{ppm}$ in the spectrum of the tung oil methyl ester (Figure 2b) corresponds to its newly formed methyl group. The absence of such peak from the spectrum of isolated $\alpha$-eleostearic acid (Figure 2c) suggests successful synthesis, purification, and drying of the desired fatty acid. As explained previously, the fatty acid is obtained in its salt form, therefore, the proton of the carboxyl group isn't detected for the fatty acid.



Figure 2. ${ }^{1} \mathrm{H}$ NMR spectra of (a) tung oil, (b) tung oil methyl esters, and (c) $\alpha$-eleostearic acid.

It is important to note that all peaks related to the fatty acid carbon backbone of tung oil (signals 1-5 and 8 , Figure 2a) are virtually unchanged after either transesterification (Figure 2b), or saponification (Figure 2c), indicating that the carbon chain is intact, including the set of three naturally conjugated carbon-carbon double bonds. Indeed, the integration of vinylic hydrogens (5.3-6.4 ppm, signal 8) with respect to the terminal methyl group $(\sim 0.8 \mathrm{ppm}$, signal 1$)$ in each spectrum confirms that the 
unsaturations were not affected by the reactions performed. In each case, the integration indicates a total of 6 vinylic hydrogens per fatty acid chain. Another indication that the unsaturations are not compromised by either transesterification or saponification of tung oil can be obtained by a quick analysis of the Raman spectra of the three samples (Figure 3). Indeed, the intense peak at approximately $1630 \mathrm{~cm}^{-1}$, associated to $C=C$ vibrations, is clearly observed in all three spectra (Figure 3).

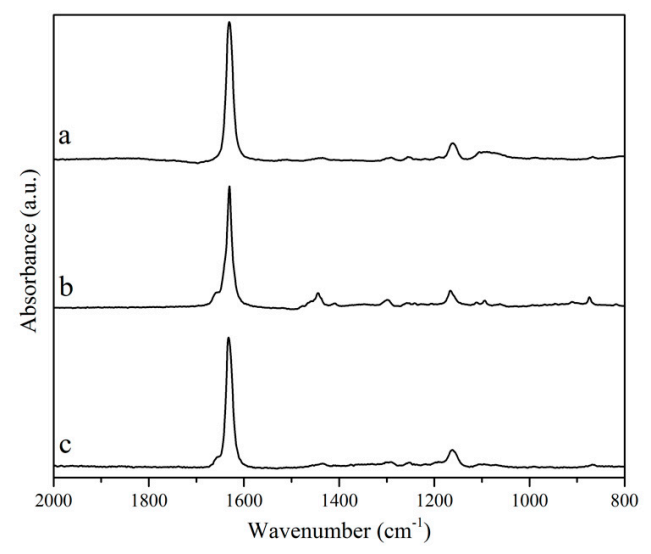

Figure 3. Raman spectra of (a) tung oil, (b) isolated $\alpha$-eleostearic acid, and (c) tung oil methyl ester.

The purity of the bio-based starting materials was verified by GC-MS. The chromatograms obtained are presented in Figure 4. In the chromatograms of tung oil and isolated $\alpha$-eleostearic acid (Figure 4a,b, respectively), two signals are observed at 15-17 min. The existence of these signals in the chromatogram of tung oil (Figure $4 \mathrm{a}$ ) is believed to relate to the various possible triglyceride structures of the oil based on its fatty acid composition ( $83 \%$ of $\alpha$-eleostearic acid, $9 \%$ of linoleic acid, $4 \%$ of oleic acid, and $4 \%-6 \%$ of palmitic acid) [21], with a predominance of triglycerides bearing $\alpha$-eleostearic acid accounting for the major signal. A similar scenario is reflected in the chromatogram of isolated $\alpha$-eleostearic acid (Figure $4 \mathrm{~b}$ ). The absence of other relevant signals suggests that, besides the mixture of fatty acids, no other by-products are detected. For the methyl esters (Figure 4c), a better separation of the different methyl ester chains is achieved, most likely due to their independent structure when compared to triglycerides, and their lower polarity in comparison to free fatty acids. Three signals are indeed obtained in the 15-17 min. range with one major signal attributed to the methyl ester of $\alpha$-eleostearic acid (Figure 4c).

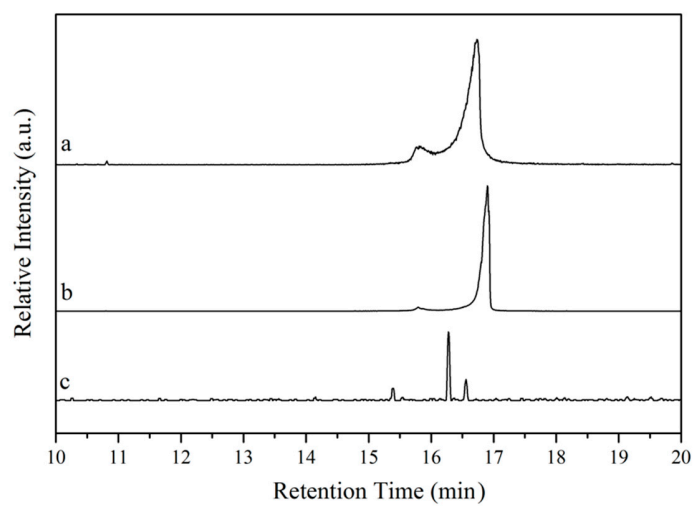

Figure 4. Chromatograms of (a) tung oil, (b) isolated $\alpha$-eleostearic acid, and (c) tung oil methyl esters.

When comparing the retention times of the major signal in each one of the chromatograms (Figure 4a-c), a clear and logical trend in retention times can be observed. On one hand, while tung oil's major signal displays a retention time of $16 \mathrm{~min}$ and $45 \mathrm{~s} \mathrm{(Figure} \mathrm{4a),} \mathrm{the} \mathrm{major} \mathrm{signal} \mathrm{for} \mathrm{the} \mathrm{fatty}$ acid sample exhibits a slightly longer retention time (16 min and $54 \mathrm{~s}$, Figure $4 \mathrm{~b}$ ) that can be attributed 
to its higher polarity and, therefore, better affinity for the GC column. On the other hand, the methyl ester's major signal exhibits a shorter retention time (16 min and $16 \mathrm{~s}$, Figure $4 \mathrm{c}$ ) in comparison to that of tung oil's major signal. Despite their similar polarities, independent methyl ester chains move more freely through the column than triglycerides, resulting in the observed shorter retention time. Based on the FT-IR, 1H NMR, Raman, and GC-MS results, it can be concluded that the preparation of methyl esters and the isolation of fatty acids from tung oil were successful.

\subsection{Cure Evaluation}

In order to establish an optimum cure schedule for the fatty acid resins prepared, the cure evolution of a crude resin containing $50 \mathrm{wt} \%$ of tung oil fatty acids, $30 \mathrm{wt} \%$ of BMA, and $20 \mathrm{wt} \%$ of DVB was monitored through dielectric analysis (DEA) at different temperatures as shown in Figure 5A, where the log of ion viscosity (an artificial property derived from the permittivity of the resin) is plotted against time at each chosen temperature. This resin composition was selected based on previous studies with similar bio-based systems [12]. The initial decrease in ion viscosity observed in the first $50 \mathrm{~min}$ of each run (Figure 5A) corresponds to the initial increase in temperature before polymerization starts. Indeed, the increase in temperature prior to extensive polymerization leads to greater molecular mobility, which translates into a decrease in ion viscosity (Figure 5A). At approximately $50 \mathrm{~min}$, the polymerization process reaches a significant rate, resulting in an increase in ion viscosity due to a decrease in molecular mobility, as the crosslinked polymer network grows. Once the ion viscosity plateaus, it is accepted that no further changes occur in the material's electrical and physical properties, indicating cure completion. When the fatty acid resin is cured at $140^{\circ} \mathrm{C}$, a significant amount of shrink cracks can be easily noticed in the sample, indicating an inappropriate cure temperature. Additionally, the DEA curve obtained at $140{ }^{\circ} \mathrm{C}$ (Figure 5A) displayed a secondary decrease in ion viscosity at approximately $12 \mathrm{~h}$, which could be attributed to exposure of the DEA sensor to air after formation of shrink cracks in the polymer, affecting the permittivity measured.

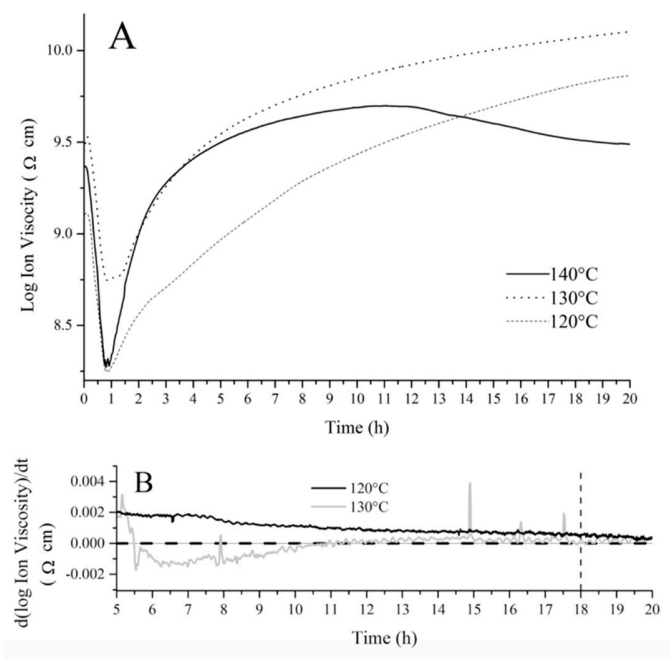

Figure 5. (A) DEA curves of fatty acid resin cured at 120, 130, and $140{ }^{\circ} \mathrm{C}$ for $20 \mathrm{~h}$; (B) Derivative of the DEA curves of the fatty acid resin cured at 130 and $120^{\circ} \mathrm{C}$ with respect to time.

For the DEA curve of the fatty acid resin cured at $120^{\circ} \mathrm{C}$ (Figure $5 \mathrm{~A}$ ), the ion viscosity continuously increases throughout the duration of the experiment $(20 \mathrm{~h})$. In order to obtain a clearer comparison between the cure at 120 and $130^{\circ} \mathrm{C}$, the derivative of ion viscosity with respect to time was plotted against cure time in Figure 5B. Close inspection of Figure 5B reveals that the derivative approaches zero (indicating the change in electrical and physical properties is zero and the cure is completed) at approximately $18 \mathrm{~h}$ (dashed line added as a guide to the eye, Figure 5B) when the resin is heated at 
$130{ }^{\circ} \mathrm{C}$. When the resin is heated at $120^{\circ} \mathrm{C}$, the derivative of the DEA curve never reaches zero for the duration of the experiment, indicating an incomplete cure after $20 \mathrm{~h}$ (Figure 5B).

A comparison of the Raman spectra of a crude fatty acid resin and a cured sample (Figure 6A) provides further evidence that cure completion was achieved for the fatty acid resin after heating at $130{ }^{\circ} \mathrm{C}$ for $18 \mathrm{~h}$. Indeed, the presence of a signal at approximately $1630 \mathrm{~cm}^{-1}$ in the spectrum of the crude resin (Figure 6A) is indicative of unreacted carbon-carbon double bonds from the various resin components. That signal is absent in the spectrum of the cured resin (Figure 6A), indicating that the polymer was fully cured as previously supported by the DEA results.
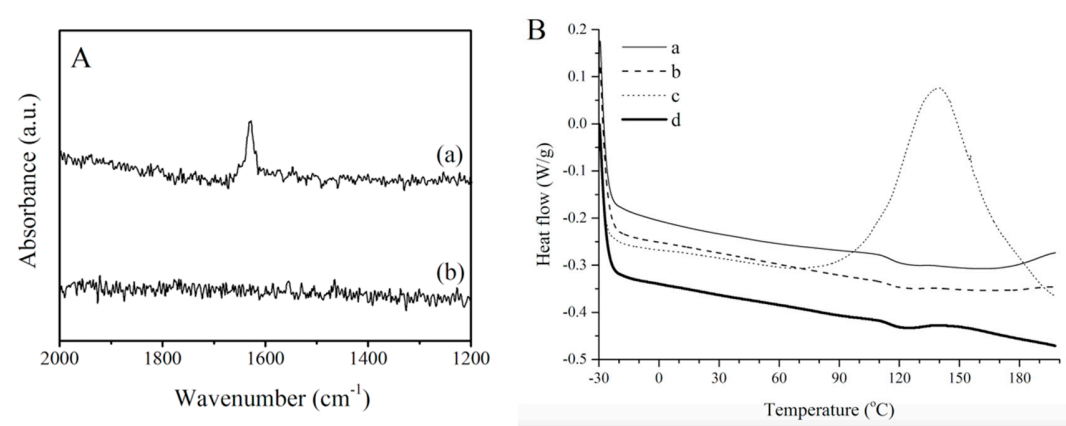

Figure 6. (A) Raman spectra of (a) tung oil fatty acid crude resin, and (b) tung oil fatty acid resin cured at $130{ }^{\circ} \mathrm{C}$ for $18 \mathrm{~h}$. (B) DSC curves of (a) tung oil resin cured at $130{ }^{\circ} \mathrm{C}$ for $18 \mathrm{~h}$, (b) tung oil fatty acid resin cured at $130^{\circ} \mathrm{C}$ for $18 \mathrm{~h}$, (c) tung oil methyl ester resin cured at $130^{\circ} \mathrm{C}$ for $18 \mathrm{~h}$, and (d) tung oil methyl ester resin cured at $130^{\circ} \mathrm{C}$ for $24 \mathrm{~h}$.

In order to validate the DEA and Raman results and to confirm cure completion, a tung oil fatty acid resin cured at $130{ }^{\circ} \mathrm{C}$ for $18 \mathrm{~h}$ was subjected to a DSC experiment (Figure 6B). The same cure schedule was applied to similar resins prepared from tung oil and from tung oil methyl esters. The DSC curves of all three samples are presented in Figure $6 \mathrm{~B}(\mathrm{a}-\mathrm{c})$. The DSC results indicate that the cure schedule used is adequate for tung oil and fatty acid resins since no exothermic peaks are observed for these two samples (Figure 6B). However, the methyl ester resin is not fully cured when subjected to that same cure schedule, as indicated by a large exothermic peak centered at approximately $140{ }^{\circ} \mathrm{C}$ (Figure 6B). In order to ensure cure completion, the tung oil methyl ester resin was subjected to a longer $(24 \mathrm{~h})$ cure schedule at $130{ }^{\circ} \mathrm{C}$. The increase in cure time by an additional $6 \mathrm{~h}$ resulted in a fully cured sample as evidenced by the absence of any exothermic peaks in the corresponding DSC curve (Figure 6B). In view of these results, the cure schedule at $130^{\circ} \mathrm{C}$ for $18 \mathrm{~h}$ is considered to be optimal for the fatty acid and tung oil resins, whereas the tung oil methyl ester resin requires heating at $130{ }^{\circ} \mathrm{C}$ for $24 \mathrm{~h}$. These cure schedules will, therefore, be employed with their corresponding resins in the remainder of this work.

\subsection{Resin Hydrophilicity}

Contact angle measurements are arguably the most widely used surface analysis technique directly related to polymer surface hydrophilicity, coating abilities, and is strongly representative of surface adhesion properties $[22,23]$. Static contact angle measurements along with their standard deviations for resins prepared with tung oil, tung oil fatty acids, and tung oil methyl esters are listed in Table 1. As anticipated, the tung oil fatty acid resin exhibited the most favorable interactions with water, reflected in the lowest contact angles measured. These results indicate an increase in the hydrophilicity of the polymer when tung oil is replaced with a more polar molecule, $\alpha$-eleostearic acid. Not surprisingly, resins prepared from tung oil and tung oil methyl esters exhibited comparable results, associated with the similar chemical structure and polarity of their main bio-based components. 
Table 1. Static and dynamic contact angles, and water absorption measurements of fully-cured resins.

\begin{tabular}{|c|c|c|c|c|c|c|c|}
\hline $\begin{array}{c}\text { Resin Bio-Based } \\
\text { Component }\end{array}$ & Static Angle $\left({ }^{\circ}\right)$ & Receding Angle $\left({ }^{\circ}\right)$ & Advancing Angle $\left({ }^{\circ}\right)$ & Hysteresis ${ }^{a}$ & $W_{w}{ }^{b}(g)$ & $W_{\mathrm{d}}{ }^{\mathrm{c}}(\mathrm{g})$ & Ratio $^{d}(\%)$ \\
\hline$\alpha$-eleostearic acid & 36.70 & 41.1 & 80.8 & 39.7 & 0.0448 & 0.0236 & 90 \\
\hline Tung oil methyl ester & 100.77 & 106.6 & 121.4 & 14.8 & 0.0395 & 0.0276 & 43 \\
\hline
\end{tabular}

${ }^{\mathrm{a}}$ Hysterisis = advancing angle - receding angle; ${ }^{\mathrm{b}} W_{\mathrm{w}}=$ wet weight; ${ }^{\mathrm{c}} W_{\mathrm{d}}=$ dry weight; ${ }^{\mathrm{d}}$ Ratio $=\frac{W_{\mathrm{W}}-W_{\mathrm{d}}}{W_{\mathrm{d}}} \times 100$.

In order to limit the interference of factors, such as surface roughness and sample heterogeneity, a dynamic analysis was also conducted (Table 1), revealing a similar trend to that of the static contact angles. The overall higher dynamic contact angles in comparison to static angles measured is most likely the result of water absorption during static measurements. Water absorption is also believed to be the reason for the high hysteresis value observed for the fatty acid resin in comparison to the other resins evaluated (Table 1). This is further supported by water uptake/absorption studies (Table 1).

Water uptake experiments were used to determine the polymer's ability to absorb water, which, consequently, can be used to make inferences about its ability to biodegrade. The ratio reflects the percentage of water absorbed/uptake. The sample prepared from fatty acids displayed $90 \%$ water uptake (Table 1), indicating favorable interaction with water and a high potential for biodegradability. The sample prepared from methyl esters also exhibited a significant amount of water uptake (43\%) despite its hydrophobic nature, displaying good potential for biodegradability. The considerably smaller water uptake observed for the sample prepared from tung oil ( $7 \%)$ can be related to its more cross-linked structure, preventing swelling and water absorption, which can significantly impact its biodegradability and recycling potential.

\subsection{Fiber-Resin Interaction}

Fatty acid, tung oil, and methyl ester resins were reinforced with $\alpha$-cellulose and the resulting composites were imaged by SEM at 2000 $\times$ and $2500 \times$ magnifications (Figure 7 ) in order to explore fiber-matrix interactions. After inspection of Figure 7A, it can be concluded that the resin prepared with tung oil fatty acids adheres well to the surface of the $\alpha$-cellulose reinforcement, as indicated by the significant amount of resin residue covering the fiber. For a composite prepared with the tung oil methyl ester resin (Figure 7B), the lack of adhesion of the resin to the reinforcement is evidenced by the clean exposed cellulose fiber. These results align well with the contact angle and water uptake data obtained, and can be considered indicative of the polarity of the resins prepared. For the composite prepared with tung oil triglycerides (Figure 7C), some resin residue can be noticed on the surface of the cellulose fiber, despite the predominant hydrophobic nature of the resin and the hydrophilic nature of cellulose. This result shows a good correlation with the static contact angle data obtained (Table 1), but at this stage, the fundamental reason for why the tung oil resin exhibits a better adhesion to the fiber's surface than the methyl ester resin is still unclear. However, it is worth noting that the intent of this work is to maximize fiber-resin interactions by preparing a novel polar tung oil fatty acid resin.

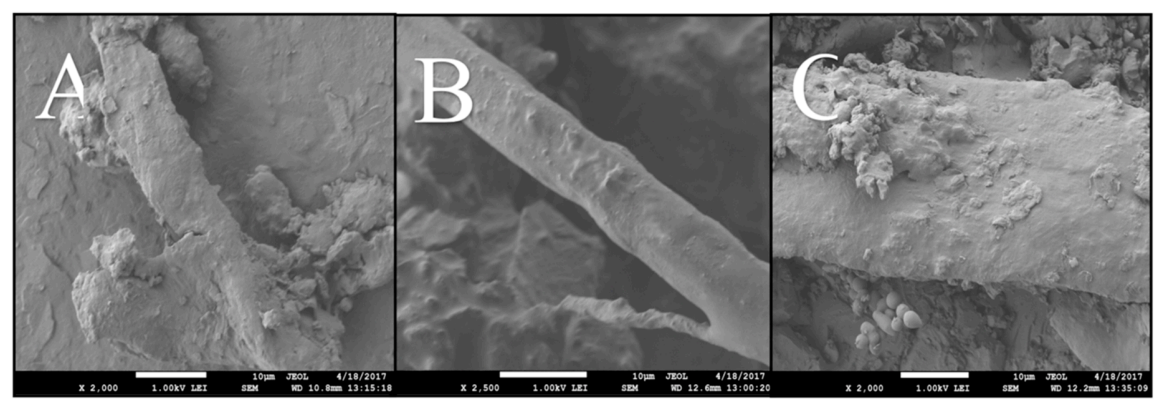

Figure 7. SEM images of (A) composite prepared with tung oil fatty acid resin, (B) composite prepared with tung oil methyl ester resin, and $(\mathbf{C})$ composite prepared with a tung oil resin. 


\subsection{Thermo-Mechanical Properties}

The thermogravimetric analysis (TGA) of tung oil, tung oil fatty acid, and tung oil methyl ester resins (Figure 8a-c) shows a clear trend for their thermal stability. It is indeed obvious that the onset degradation temperature ( $\left.T_{\text {onset }}\right)$ for the tung oil methyl ester resin $\left(\sim 100^{\circ} \mathrm{C}\right)$ is much lower than that of samples prepared with tung oil fatty acids $\left(\sim 180^{\circ} \mathrm{C}\right)$ or tung oil triglycerides $\left(\sim 300^{\circ} \mathrm{C}\right)$. This trend can be explained as a consequence of the chemical structure of the major resin components. For example, the markedly higher $T_{\text {onset }}$ observed for the tung oil resin is the result of a highly crosslinked structure, due to the fact that the tung oil triglyceride monomer consists of three fatty acid esters connected to a glycerol backbone. In both fatty acids and methyl esters, the polymerizable fatty chains are independent from each other, resulting in a much less crosslinked polymer network. The difference observed in the $T_{\text {onset }}$ of fatty acid and methyl ester polymers can be associated to their polarity. The polar fatty acid resin exhibits stronger hydrogen bonding interactions between polymer chains, resulting in a more compact arrangement of the overall polymer structure and, therefore, constituting a more thermally stable material in comparison to its methyl ester analogue.
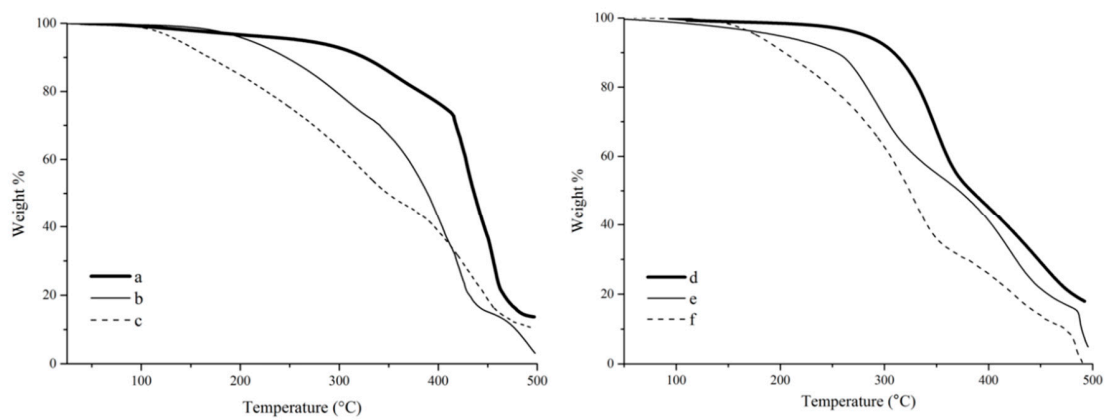

Figure 8. TGA curves of (a) tung oil resin, (b) tung oil fatty acid resin, (c) tung oil methyl ester resin, (d) composite prepared with tung oil resin, (e) composite prepared with tung oil fatty acid resin, and (f) composite prepared with tung oil methyl ester resin.

It is also apparent, from the TGA results (Figure 8a-c), that the bulk of the thermal degradation process occurs from $T_{\text {onset }}$ to approximately $480^{\circ} \mathrm{C}$ for the resins prepared with tung oil and methyl esters (Figure $8 \mathrm{a}, \mathrm{c}$ ), while the resin prepared with fatty acids clearly exhibits an additional secondary step starting at approximately $450{ }^{\circ} \mathrm{C}$ (Figure $8 \mathrm{~b}$ ). The major degradation process is believed to correspond to the breakdown of the cross-link network of the thermoset. The additional degradation step observed for the resin prepared with fatty acids could be associated to the final oxidation of residues and char formation, but its definitive origin can't be reliably inferred from the TGA results alone, lying outside the scope of the work described in this manuscript.

A very similar trend in thermal stability can be observed for the composites (Figure $8 \mathrm{~d}-\mathrm{f}$ ) compared to their corresponding resins (Figure 8a-c). All composites exhibit a very clear thermal degradation profile, with two distinct stages that can be associated with the degradation of the resin and the degradation of the reinforcement, although it is unavoidable that the degradation of these two components (resin and reinforcement) occur with some overlap. When correlating the results observed with fiber-matrix interactions, one would expect that a stronger binding between resin and reinforcement would result in a better fiber protection, and ultimately in a delayed degradation, especially towards the end of the thermal degradation process. Indeed, when comparing the temperature at $80 \mathrm{wt} \%$ degradation $\left(T_{80}\right)$ for resins and their corresponding composites, respectively, an increase from $\sim 430$ to $\sim 450{ }^{\circ} \mathrm{C}$ can be seen for samples prepared with fatty acids, whereas a decrease from $\sim 445$ to $\sim 425{ }^{\circ} \mathrm{C}$ is observed for samples prepared with methyl esters. This behavior is reflective of the better fiber-matrix interactions obtained with fatty acids in comparison to methyl esters. In the case of tung oil triglyceride, the increase in $T_{80}$ from $\sim 455$ to $\sim 475^{\circ} \mathrm{C}$, for resin and composite, respectively, can be associated to the 
highly crosslinked structure of the oil offering thermal protection to the fiber despite the expected poor fiber-matrix interaction.

DMA was used to assess thermo-mechanical properties of the resins and composites studied. These results are summarized in Figure 9 and Table 2. At temperatures below $20^{\circ} \mathrm{C}$, the resin prepared with fatty acids displays a higher storage modulus than the resin prepared with tung oil triglycerides (Figure 9a,b), exhibiting a behavior that is reflective of the expected, more prevalent hydrogen bonding. Above $20^{\circ} \mathrm{C}$, it is believed that chain mobility overcomes hydrogen bonding, and that the more crosslinked structure of the resin prepared with tung oil accounts for the higher storage modulus observed in comparison to the resin prepared with fatty acids. At the rubbery plateau $\left(T_{\mathrm{g}}+50^{\circ} \mathrm{C}\right)$, above all phase transitions of the materials, the storage modulus for the resin prepared with tung oil is approximately 4.7 times higher than that of the resin prepared with fatty acids (68.9 MPa vs. 14.6 $\mathrm{MPa}$, respectively, Table 2). Indeed, that same trend is also clear in the calculated crosslink density, with $16.2 \times 10^{-4} \mathrm{~mol} / \mathrm{cm}^{3}$ for the fatty acid resin and $77.7 \times 10^{-4} \mathrm{~mol} / \mathrm{cm}^{3}$ for the tung oil resin (Table 2). Cross-link densities were calculated based on the rubbery elasticity theory, according to Equation (1) [24,25]:

$$
\text { Crosslink density }=E^{\prime} / R T
$$

where $E^{\prime}$ is the storage modulus at $T_{\mathrm{g}}+50^{\circ} \mathrm{C}, \mathrm{R}$ is the gas constant, and $T$ is the absolute temperature (K). Crosslink density was not calculated for composites (Table 2) because the numbers would be artificially inflated by the natural increase in $E^{\prime}$ resulting from the addition of a reinforcement, therefore masking the true crosslink density value. It is also worth noting that DMA specimens could not be produced from the resin prepared with methyl esters due to its exceeding fragility.

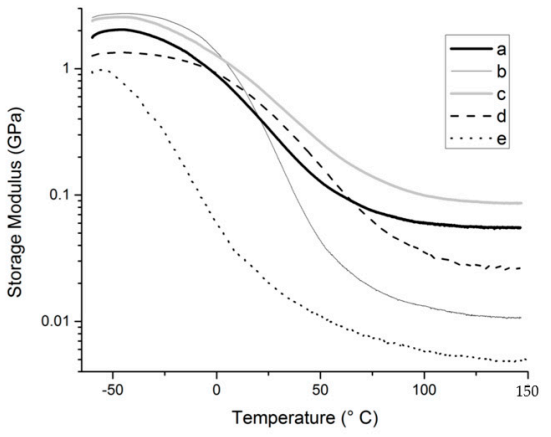

(A)

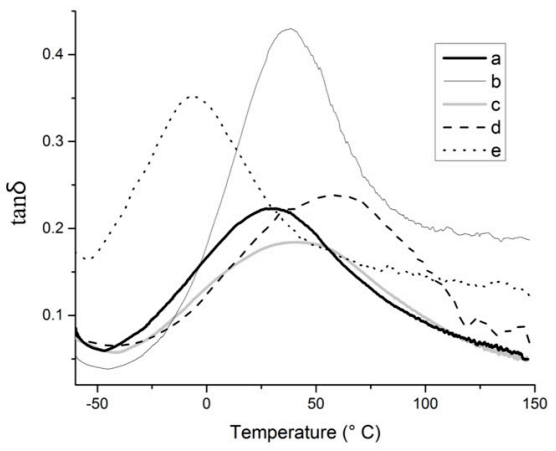

(B)

Figure 9. (A) Storage modulus (E') vs. temperature and (B) tan $\delta$ curves for (a) a resin prepared with tung oil, (b) a resin prepared with fatty acids, (c) a composite prepared with tung oil, (d) a composite prepared with fatty acids, and (e) a composite prepared with methyl esters.

Table 2. DMA results for samples prepared with tung oil, tung oil fatty acids, and tung oil methyl esters.

\begin{tabular}{cccccc}
\hline $\begin{array}{c}\text { Major Resin } \\
\text { Component }\end{array}$ & Reinforcement & $\mathbf{T}_{\mathbf{g}}{ }^{\mathbf{a}}\left({ }^{\circ} \mathbf{C}\right)$ & $\begin{array}{c}\boldsymbol{E}^{\prime} \text { at } \mathbf{2 5}{ }^{\circ} \mathbf{C} \\
\mathbf{( M P a )}\end{array}$ & $\begin{array}{c}\boldsymbol{E}^{\prime} \text { at } \boldsymbol{T}_{\mathbf{g}}+\mathbf{5 0} \\
\left.{ }^{\circ} \mathbf{C} \mathbf{( M P a}\right)\end{array}$ & $\begin{array}{c}\text { Cross Link Density } \\
\left(\frac{\mathbf{m o l}}{\mathbf{c m}^{3}} \times 10^{-4}\right)\end{array}$ \\
\hline Tung oil & - & 30 & 338.4 & 68.9 & 77.7 \\
Fatty acid & - & 39 & 284.8 & 14.6 & 16.2 \\
Tung oil & $\alpha$-cellulose & 38 & 524.0 & 131.2 & - \\
Fatty acid & $\alpha$-cellulose & 56 & 459.5 & 31.7 & - \\
Methyl ester & $\alpha$-cellulose & -6 & 20.2 & 11.8 & - \\
\hline \multicolumn{7}{c}{$T_{g}$ was obtained from the peak of the tan $\delta$ curve. }
\end{tabular}

In the presence of the cellulose reinforcement, the composite prepared with methyl esters exhibits significant lower storage modulus than the composite made from fatty acids (Figure 9d,e), indicating that a stronger reinforcing effect is obtained for the more polar resin, as expected due to its increased affinity with the reinforcement. For the composite prepared with tung oil triglyceride (Figure 9c), the storage modulus is consistently higher than all other composites, revealing that, contrary to 
expected, the crosslink density of the resin is a greater contributor towards final mechanical properties than fiber-matrix interactions. It is interesting to note that at lower temperatures, the fatty acid resin (Figure 9b) exhibits higher storage modulus than the other systems, surpassing the tung oil resin (Figure 9a) below $24^{\circ} \mathrm{C}$ as discussed previously, the fatty acid composite (Figure $9 \mathrm{~d}$ ) below $23^{\circ} \mathrm{C}$, and the tung oil composite (Figure 9c) below $3{ }^{\circ} \mathrm{C}$. This trend indicates that below the $T_{\mathrm{g}}$ of the polar resin, hydrogen bonding between the resin and reinforcement is limited, therefore, the ligno-cellulosic filler particles represent weak points within the sample, contributing to the lower mechanical performance observed at lower temperatures in comparison to the pure resin.

For all samples investigated, the $\tan \delta$ curves (Figure 9) exhibit a single major peak, representing the glass transition of the system evaluated. The presence of a single transition is indicative of a homogeneous polymer with no evident phase separation. It is worth noting that the use of vegetable oils with less carbon-carbon double bonds typically results in polymers with more than one $T_{\mathrm{g}}[8,9]$, and this behavior has been attributed to a micro-phase separation within the polymer network due to a significant difference in reactivity of the co-monomers used. The presence of a single tan $\delta$ peak for the composite samples (Figure $9 \mathrm{c}-\mathrm{e}$ ) also suggests that cellulose fibers have been evenly distributed throughout the polymer, providing universal thermo-mechanical properties for the entire sample. Another indicator of resin heterogeneity is the breadth of the tan $\delta$ peak. Indeed, the narrower the peak, the more homogeneous the system. It is interesting to notice how the addition of reinforcement changes the breadth of the $\tan \delta$ peak for the samples prepared with fatty acids (Figure $9 \mathrm{~b}, \mathrm{~d}$ ). The same effect is not observed for the other resins, therefore revealing greater interaction between resin and reinforcement on the composite prepared with fatty acids. Indeed, as observed previously in similar systems, a greater interaction of one of the resin components with the reinforcement contributes to composite heterogeneity at the molecular level [8].

The greater interaction between fatty acids and cellulose is also reflected in the $T_{\mathrm{g}}$ values of the samples (Table 2). Indeed, there is only a small change in the $T_{\mathrm{g}}$ of samples prepared with tung oil upon addition of cellulose, revealing that the presence of cellulose has little effect on the polymerization of the resin and its intrinsic properties. For the fatty acid resin, a significant increase of the $T_{\mathrm{g}}$ from 39 to $56^{\circ} \mathrm{C}$ (Table 2) is observed upon the addition of cellulose. This increase in $T_{\mathrm{g}}$ suggests a stronger affinity between resin and reinforcement, resulting in more restricted chain mobility. Among all samples, the composite prepared with methyl esters exhibits the lowest $T_{\mathrm{g}}\left(-6^{\circ} \mathrm{C}\right.$, Table 2$)$, indicating the combination of a weak resin with a poor adhesion between the resin and reinforcement.

Finally, the maximum value in the $\tan \delta$ curve $\left(\tan \delta_{\max }\right)$ can be associated to the damping properties of the material. It is noteworthy that all composites exhibit $\tan \delta_{\max }$ values lower than their corresponding unreinforced resins (Figure 9), with a more pronounced decrease (from approximately 0.44 to approximately 0.23 , Figure $9 b, d$ ) for the fatty acid system. This greater decrease is most likely the result of limited ability of the composite in dispersing shock/sound waves into molecular/chain movements, due to the greater interaction between resin and reinforcement, effectively interlocking the polymer chains through hydrogen bonding. When comparing the different composites, it is clear that the sample prepared with methyl esters exhibits the highest $\tan \delta_{\max }$, which can be associated to a better ability to dampen vibration through dissipation of the energy into polymer chain movement.

\section{Conclusions}

In conclusion, this manuscript discusses the successful isolation and purification of $\alpha$-eleostearic acid from tung oil via saponification, and its subsequent co-polymerization with DVB and BMA, with the purpose of preparing bio-based composites with enhanced resin-reinforcement interactions. After optimization of the novel fatty acid-based system and establishment of an appropriate cure schedule, a comparison of the morphology, hydrophilicity, and thermo-mechanical properties of cellulose-reinforced composites prepared with $\alpha$-eleostearic acid, tung oil triglycerides, and tung oil methyl esters revealed trends that can be associated to resin-reinforcement interactions and the resin's crosslink density. SEM imaging indicated stronger resin-reinforcement interactions for composites 
prepared with $\alpha$-eleostearic acid, confirming the trends obtained with the contact angle experiments, which suggested that the fatty acid resin exhibits the most favorable interactions with water due to its higher polarity in comparison to tung oil and tung oil methyl esters.

From the thermo-mechanical analyses performed (TGA and DMA), it was observed that the tung oil resin had superior properties due to its higher crosslink density, whereas samples prepared with tung oil methyl esters displayed the worst thermo-mechanical properties, as expected based on its lower crosslink density, lower polarity and, therefore, poor compatibility with the reinforcement. The results presented herein indicate the viability of enhancing resin-reinforcement interactions at the cost of crosslink density. Such an approach can find use in applications that require high water absorption, swelling, and degradability, such as naturally decomposable flower pots, for example.

Supplementary Materials: The following are available online at http://www.mdpi.com/2079-6412/9/7/447/s1, Figure S1: Reaction mechanism for the free radical polymerization of $\alpha$-eleostearic acid with di-tert butyl peroxide (DTBP) as the free radical initiator, Figure S2: General schematic for the preparation of thermosetting composites.

Author Contributions: Conceptualization, Writing-Review and Editing, Supervision, and Funding Acquisition, R.L.Q.; Methodology and Writing-Original Draft Preparation, A.M.

Funding: This research was funded by Herty Advanced Materials Development Center through the 2016 Herty Fellowship.

Conflicts of Interest: The authors declare no conflict of interest.

\section{References}

1. Yuan, H.; Yang, B.; Zhu, G. Biodiesel production with water-tolerance and microwave absorbing catalyst using tung oil. Int. J. Green Energy 2013, 10, 999-1010. [CrossRef]

2. Mosiewicki, M.A.; Casado, U.; Marcovich, N.E.; Aranguren, M.I. Polyurethanes from tung oil: Polymer characterization and composites. Polym. Eng. Sci. 2009, 49, 685-692. [CrossRef]

3. Ashraf, S.M.; Ahmad, S.; Riaz, U. Development of novel conducting composites of linseed-oil-based poly(urethane amide) with nanostructured poly(1-naphthylamine). Polym. Int. 2007, 56, 1173-1181. [CrossRef]

4. Sharma, H.O.; Alam, M.; Riaz, U.; Ahmad, S.; Ashraf, S.M. Miscibility studies of polyesteramides of linseed oil and dehydrated castor oil with poly(vinyl alcohol). Int. J. Polym. Mater. 2007, 56, 437-451. [CrossRef]

5. Zhan, M.; Wool, R.P. Biobased composite resins design for electronic materials. J. Appl. Polym. Sci. 2010, 118, 3274-3283. [CrossRef]

6. Biermann, U.; Butte, Q.; Holtgrefe, R.; Feder, W.; Metzger, J.O. Esters of calendula oil and tung oil as reactive diluents for alkyd resins. Eur. J. Lipid Sci. Technol. 2010, 112, 103-109. [CrossRef]

7. Das, G.; Karak, N. Thermostable and flame retardant Mesua ferrea L. seed oil based non-halogenated epoxy resin/clay nanocomposites. Prog. Org. Coat. 2010, 69, 495-503. [CrossRef]

8. Quirino, R.L.; Larock, R.C. Rice hull biocomposites, part 2: Effect of the resin composition on the properties of the composite. J. Appl. Polym. Sci. 2011, 121, 2050-2095. [CrossRef]

9. Quirino, R.L.; Woodford, J.; Larock, R.C. Soybean and linseed oil-based composites reinforced with wood flour and wood fibers. J. Appl. Polym. Sci. 2012, 124, 1520-1528. [CrossRef]

10. Nozawa, A.; Nanamiya, H.; Tozawa, Y. Production of membrane proteins through the wheat-germ cell-free technology. In Methods in Molecular Biology; Humana Press: Clifton, NJ, USA, 2010; pp. 213-218.

11. Park, K.; Berrier, C.; Lebaupain, F.; Pucci, B.; Popot, J.; Ghazi, A.; Zito, F. Fluorinated and hemifluorinated surfactants as alternatives to detergents for membrane protein cell-free synthesis. Biochem. J. 2007, 403, 183-187. [CrossRef]

12. Johns, A.; Morris, S.; Edwards, K.; Quirino, R.L. Asolectin from soybeans as a natural compatibilizer for cellulose-reinforced biocomposites from tung oil. J. Appl. Polym. Sci. 2015, 132, 41833. [CrossRef]

13. Salimon, J.; Abdullah, B.M.; Salih, N. Saponification of Jatropha curcas seed oil: Optimization by D-optimal design. Int. J. Chem. Eng. 2012, 2012, 574780. [CrossRef]

14. Liu, C.; Yang, X.; Cui, J.; Zhou, Y.; Hu, L.; Zhang, M.; Liu, H. Tung oil based monomer for thermosetting polymers: Synthesis, characterization, and copolymerization with styrene. Bioresources 2012, 7, 0447-0463. 
15. Kaufman, M.; Wiesman, Z. Pomegranate oil analysis with emphasis on MALDI-TOF/MS triacylglycerol fingerprinting. J. Agric. Food Chem. 2007, 55, 10405-10413. [CrossRef] [PubMed]

16. Pfister, D.P.; Baker, J.R.; Henna, P.H.; Lu, Y.; Larock, R.C. Preparation and properties of tung oil-based composites using spent germ as a natural filler. J. Appl. Polym. Sci. 2008, 108, 3618-3625. [CrossRef]

17. Hondred, P.R.; Salat, L.; Mangler, J.; Kessler, M.R. Tung oil-based thermosetting polymers for self-healing application. J. Appl. Polym. Sci. 2014, 131, 40406. [CrossRef]

18. Klemm, D.; Heublein, B.; Fink, H.; Bohn, A. Cellulose: Fascinating biopolymer and sustainable raw material. Angew. Chem. Int. Ed. Engl. 2005, 44, 3358-3393. [CrossRef]

19. Quirino, R.L.; Larock, R.C. Rh-based biphasic isomerization of carbon-carbondouble bonds in natural oils. J. Am. Oil Chem. Soc. 2012, 89, 1113-1124. [CrossRef]

20. Schönemann, A.; Edwards, H.G.M. Raman and FTIR microspectroscopic study of the alteration of Chinese tung oil and related drying oils during ageing. Anal. Bioanal. Chem. 2011, 400, 1173-1180. [CrossRef]

21. Quirino, R.L.; Larock, R.C. Bioplastics, biocomposites, and biocoatings from natural oils. In Renewable and Sustainable Polymers; Payne, G.F., Smith, P.B., Eds.; ACS Publications: Washington, DC, USA, 2011; pp. $37-59$.

22. Belibel, R.; Barbaud, C.; Mora, L. Dynamic contact angle cycling homogenizesheterogeneous surfaces. Mater. Sci. Eng. C Mater. Biol. Appl. 2016, 69, 1192-1200. [CrossRef]

23. Strobel, M.; Kirk, S.M.; Heinzen, L.; Mischke, E.; Lyons, C.S.; Endle, J.; Dillingham, G. Contact angle measurements on oxidized polymer surfaces containing water-soluble species. J. Adhes. Sci. Technol. 2015, 29, 1483-1507. [CrossRef]

24. Flory, P.J. Principles of Polymer Chemistry; Cornell University Press: Ithaca, NY, USA, 1953.

25. Ward, I.M. Mechanical Properties of Solid Polymers; Wiley Interscience: New York, NY, USA, 1971.

(C) 2019 by the authors. Licensee MDPI, Basel, Switzerland. This article is an open access article distributed under the terms and conditions of the Creative Commons Attribution (CC BY) license (http://creativecommons.org/licenses/by/4.0/). 